\title{
De Humboldt a Darwin: una inflexión clave en la historia de la biogeografía
}

\author{
Gustavo Caponi $^{*}$
}

\section{Resumen}

Comparada con la biogeografía humboldtiana, las tesis biogeográficas que Darwin desarrolla en El Origen de las Especies presentan dos novedades importantes. La primera de ella es teórica: para Darwin la distribución actual de los seres vivos está más directamente determinada por factores bióticos que por clima y el suelo. La segunda diferencia, en cambio, es más de carácter metodológico: la biogeografía de Humboldt se niega a considerar los factores históricos que podrían explicar esa distribución. No sólo la idea de una vinculación genealógica entre las especies está ausente del punto de vista humboldtiano; sino que hasta la historia de la tierra es ignorada como factor explicativo relevante para entender la distribución actual de los seres vivos.

Palabra Claves: Biogeografia; Buffon, Darwin, C.; De Candolle, A.; Humboldt, A.; Lyell, C..

\begin{abstract}
If compared with Humboldt's Biogeography, the Biogeographical theses that Darwin develops in The Origin of the Species present two important new features. The first is theoretical: for Darwin the actual distribution of the living beings is more directly determined by biotic factors than by climate and soil. The

* Departamento de Filosofia, Universidade Federal de Santa Catarina (caponi@cfh.ufsc.br).
\end{abstract}

Geosul, Florianópolis, v. 23, n. 45, p 27-41, jan./jun. 2008 
CAPONI, G. De Humboldt a Darwin: una inflexión clave en la historia ...

second difference, however, is rather methodological: the Biogeography of Humboldt doesn't consider the historical factors that could explain that distribution. Not only the idea of a genealogical relationship between the species is absent in the Humboldt's point of view; but also the History of the Earth it is ignored like an important explanatory factor to understand the actual distribution of living beings.

Key words: Biogeography; Buffon, Darwin, C.; De Candolle, A.; Forbes, E.; Humboldt, A.; Lyell, C..

Ya en el inicio de El Origen de las Especies, y contrariando el punto de vista de naturalistas tan influyentes como Buffon (1749, p.528) y Cuvier (1798, p.10), Darwin $(1859$, p.3) afirmaba que si se consideraba a las "condiciones externas tales como clima, comida etc., como si fuesen las únicas causas de variación", no se conseguía explicar cosas tales como "la estructura del pájaro carpintero, con sus pies, cola y lengua tan admirablemente adaptadas a capturar insectos bajo la corteza de los árboles". Para él era evidente que los diferentes perfiles de cada ser vivo estaban indisolublemente vinculados con las estructuras "de todos los otros seres orgánicos con los cuales (él) entra en competición por comida o residencia, o de los cuales tiene que escapar o a los cuales tiene que apresar"(Darwin, 1859, p.77); y por eso era necesario determinar cuál era el mecanismo natural que producía esa adaptaciones definitivamente inexplicables por la mediación de procesos fisiológicos como aquellos que la alimentación y el clima pueden desencadenar en los organismos individuales.

Logro éste que sólo sería posible yendo más allá de una biología de causas próximas que actúan en y sobre el organismo individual, y accediendo al plano de una biología de causas remotas que actúan en y sobre las poblaciones. Sería sólo en ese dominio epistemológico, ignorado por toda la historia natural anterior a Darwin, en donde la teoría de la selección natural podría ser formulada (cfr. Caponi, 2006, pp.27-28). Pero no era sólo en las 
CAPONI, G. De Humboldt a Darwin: una inflexión clave en la historia ...

singularidades morfológicas de los seres vivos en donde Darwin encontraría elementos que lo llevarían a comprender que "la relación biológica fundamental es la relación entre el viviente y otros vivientes" y que ésta "supera a la relación entre el viviente y el medio, concebido como conjunto de fuerzas físicas"(Canguilhem, 1965, p.137): el otro gran conjunto de evidencias a favor de esa conclusión vendría de los hechos relativos a la distribución geográfica de los seres vivos.

En consonancia con la idea de que "el primer medio en el cual vive un organismo, es un entorno de vivientes que para él son sus enemigos o aliados, presas o depredadores" (Canguilhem, 1965, p.137), Darwin (1859, p.140) iría a sostener "que las especies en estado de naturaleza están estrictamente limitadas a sus áreas por la competencia de otros seres orgánicos, tanto más que por la adaptación a sus climas particulares"; y esta posición no sólo era claramente contraria a la posición de Linneo, sino que también implicaba un distanciamiento significativo con relación a la biogeografía de inspiración buffoniana sustentada por una figura tan influyente y respetada como la de Alexander von Humboldt.

Claro, ni Buffon, ni Humboldt, adherían ya a la estricta correlación entre el clima y la distribución de los seres vivos que, por lo menos para el caso de los vegetales, parece sugerida en el Discurso sobre el aumento de la tierra habitable de Linneo ([1744]2001, §45 a §53). Buffon (1761, p.96) había establecido que los animales de la zona tórrida del Nuevo Mundo no eran los mismos que los de la zona tórrida del Viejo Mundo; y Humboldt (1805, p.22) demostró que en el caso de los vegetales también se cumplía esa falta de correlación entre el clima y la distribución geográfica de las diferentes especies. Pero, pese al reconocimiento de que los factores climáticos eran insuficientes para explicar la distribución actual de los seres vivos, ambos naturalistas continuaron pensando que el clima y el suelo eran las causas más inmediatas de esa distribución; y ambos tendieron a ignorar o a menospreciar los factores puramente bióticos que luego Darwin iba a apuntar como fundamentales para comprenderla. Para ellos: las 
CAPONI, G. De Humboldt a Darwin: una inflexión clave en la historia ...

semejanzas de clima y de suelo entre dos regiones ya no garantizaban la semejanza o identidad de las especies biológicas que las ocupaban; pero las diferencias de clima y de suelo eran consideradas como causas en principio suficientes para explicar la diversidad biológica.

Para Buffon, en efecto, la relación entre el clima y la distribución geográfica de los animales continua siendo muy estrecha y nítida (cfr. Flourens,1850, pp.152-154; Roger,1989, pp.390-399). Para él, todo animal "tiene su país, su patria natural, en la cual cada uno es retenido por necesidad física; cada uno es hijo de la tierra que habita, y es en ese sentido que se debe decir que tal o cual animal es originario de tal o cual clima"(Buffon, 1761, p.2). Por eso, nos dice Buffon $(1761$, p.2) algunas especies "solamente pueden propagarse en los países cálidos"; y otras "solo pueden subsistir en los climas fríos: "el león jamás habitó las regiones del norte; y el reno no se encuentra nunca en las regiones meridionales".

Que el Lobo Guará sólo exista en Sudamérica sería un hecho que Buffon atribuiría a procesos que tendrían que ver con la historia de la tierra (cfr. Buffon: 1761, p.127 y 1766, p.373). Pero que dentro de ese continente ese animal sea encontrado sólo en el Paraguay, en el sur de Brasil y en el nordeste de Argentina, y no, por ejemplo, en las zonas templadas y frías de este último país, es algo que simplemente se explica por una cuestión climática: el Lobo Guará es un animal de clima cálido y húmedo; por eso no desciende hacia las praderas pampeanas aun cuando ninguna barrera geográfica se lo impida. Y esa sería más o menos la misma línea de raciocinio que Humboldt seguiría para explicar la distribución de una especie vegetal en el interior de una misma área continental en la que no existan barreras geográficas que puedan limitar su diseminación.

Humboldt sabía, en efecto, que las plantas de la zona fría de un continente podían no ser las mismas de la zona fría de otro continente; pero aun así intentó correlacionar la distribución geográfica de esas plantas con bandas isotérmicas e isobáricas que 
CAPONI, G. De Humboldt a Darwin: una inflexión clave en la historia ...

variaban conforme la latitud y altitud de las diferentes regiones (Humboldt, [1845]1977, p.72). Desde su punto de vista, nos dice Peter Bowler (1998, p.198):

"Había una equivalencia entre las fajas de vegetación encontradas a diferentes alturas al ascender una montaña y las zonas geográficas que circundan la tierra. Las plantas de las grandes alturas eran equivalentes de la flora del ártico, ambas adaptadas a condiciones de frío. Las zonas de vegetación en torno de la tierra estaban determinadas por la temperatura y la precipitación pluvial, y dentro de cada zona todas las plantas se habían adaptado a las condiciones de manera similar.

Claro, las especies "no eran las mismas: continentes diferentes estaban habitados por especies diferentes adaptadas a los mismos conjuntos de condiciones" y por eso "la existencia de provincias geográficas distintas no podía ser explicada en función del clima"(Bowler, 1998, p.198). Pero, sin ser idénticas, esas especies podían ser descriptas como "análogas de aspecto y de fisonomía”(Humboldt, [1845]1977, p.44); y esa semejanza sí era explicable por el factor climático: del mismo modo en que, para Buffon (1761, pp.102-103) o Isidore Geoffroy Saint-Hilaire (1832, pp.569-570), condiciones climáticas similares actuando sobre especies semejantes podían redundar en alteraciones morfológicas análogas; para Humboldt, la similitud climática entre dos regiones podía permitir explicar la semejanza de conformación, aunque no la identidad, de las especies que en ella habitaban. Su foco de análisis, sin embargo, no estaba tanto en la distribución de especies particulares sino en la distribución de asociaciones vegetales; es decir, formas de agrupamiento entre diferentes tipos de especies:

Como Tournefort en el Ararat, Humboldt demostró que en el Chimborazo había una sucesión altitudinal de floras; pero, lo más importante fue que también mostró que esa sucesión altitudinal era muy semejante o análoga a la sucesión latitudinal que se observaba en la superficie de la tierra. Cada zona de vegetación en las laderas del 
CAPONI, G. De Humboldt a Darwin: una inflexión clave en la historia ...

Chimborazo poseía una 'fisonomía propia'; y cada una de ellas podía ser comparada a una zona latitudinal existente en la superficie del globo. Humboldt llegó a distinguir quince conjuntos diferentes de asociaciones vegetales en la superficie terrestre: tundras, florestas de coníferas, praderas etc. Cada uno de esos conjuntos se caracterizaba por la diferente proporción de las principales clases y órdenes de vegetales y por una correspondencia notable con ciertas características ambientales. La correlación entre formación vegetal y ambiente era tan íntima y tan característica que, por el estudio de especies particulares, o por la diferente proporción de sus constituyentes, se podía, a partir de una determinada formación vegetal, hacer inferencias sobre las características físicas del ambiente. El mundo podía ser, por lo tanto, mapeado en formaciones vegetales y éstas relacionadas a características tales como presión atmosférica, temperatura, humedad etc. Para posibilitar a delimitación precisa de esas características físicas que delimitaban las formaciones vegetales en el espacio, Humboldt inventó las 'isobaras', líneas que unen puntos con la misma presión atmosférica, las 'isotermas', que unen puntos con la misma temperatura y así por delante. Por el trazado y comparación de varias de esas líneas isométricas era posible determinar cuáles eran los factores ambientales que delimitaban las diferentes formaciones vegetales. (Papavero// Teixeira//Llorente-Bousquets, 1997, p.183).

La temperatura, como podemos ver, no era ciertamente el único factor climático considerado por Humboldt: para él, otros factores físicos tales como la presión atmosférica, la humedad y la tensión eléctrica también incidían en la distribución de los vegetales (Humboldt, 1805, p.14; Drouin,1991, p.68). No cabe duda, sin embargo, que, desde su punto de vista, la temperatura jugaba ahí un papel central. En su opinión, "a pesar de la influencia que la presión del aire y la extinción más o menos grande de la luz ejercen sobre las funciones vitales de las plantas, es con todo el calor desigualmente distribuido entre las distintas partes del año, lo que ha de considerarse como el estímulo más poderoso de la 
CAPONI, G. De Humboldt a Darwin: una inflexión clave en la historia ...

vegetación"(Humboldt, [1802]1991a, p. 346). Por otro lado, si bien es cierto que esa asociación entre el clima y la distribución geográfica de los seres vivos se refiere fundamentalmente a las plantas, tampoco deja de ser verdad que, aunque con algunas dudas o restricciones, Humboldt también pretendía extender esa correlación a los animales (cfr. Humboldt, [1845]1977, p.71).

Éstos, según leemos en su Ensayo sobre la Historia Natural del Cóndor, también "siguen, aunque ciertamente menos que las plantas, esa identidad de formas en sitios que están alejados los unos de los otros, pero que gozan de un clima análogo"; y esta suposición hace que Humboldt llegue a plantearse la siguiente pregunta: " $¿ \mathrm{Si}$, en el medio de las inmensas planicies del valle del Amazonas, una montaña aislada se elevase hasta las regiones heladas, esa montaña estaría habitada por cóndores, guanacos o vicuñas?"(Humboldt, 1811, p.38). Prudente, Humboldt deja la cuestión sin responder porque de hecho no cuenta con los datos necesarios para hacerlo: sus observaciones zoogeográficas eran de hecho mucho menos precisas y completas que sus observaciones fitogeográficas. Pero es evidente, sin embargo, que la cuestión sólo fue planteada para sugerir la posibilidad de que esa correlación entre clima y fauna efectivamente se cumpla por lo menos con cierta aproximación.

Es menester reconocer, por otro lado, que un observador tan agudo como Humboldt no podía dejar de percibir que, en algunas ocasiones por lo menos, las variables climáticas eran insuficientes para explicar la distribución geográfica de los seres vivos al interior de una misma área continental. Tal es el caso de lo que ocurría con la distribución geográfica de los molestos y omnipresentes mosquitos: la misma, según también leemos en el Viaje a las Regiones Equinocciales del Nuevo Mundo, no parece "depender únicamente del calor del clima, del exceso de humedad o de lo cuajado de las selvas, sino de circunstancias locales difíciles de precisar"(Humboldt, [1802]1991b,p.69); y esas circunstancias, podría decir Darwin, sólo serían dificiles de precisar porque no se le estaría dando la debida atención a los 
CAPONI, G. De Humboldt a Darwin: una inflexión clave en la historia ...

factores específicamente biológicos que estarían incidiendo en dicha distribución (cfr. Darwin,1859, p.139).

Para Darwin (1859, p.175), en efecto, quienes consideraban "el clima y las condiciones físicas de vida como los elementos esencialmente importantes de distribución de los seres orgánicos", cometían dos errores. El primero consistía en sobrestimar "el grado de adaptación de las especies a los climas en que viven". El segundo consistía en ignorar que "el área ocupada por los individuos de una especie en un país cualquiera no depende de modo alguno exclusivamente del cambio gradual e insensible de las condiciones físicas, sino que depende, en gran parte, de la presencia de otras especies a costa de las cuales vive, o por las que es destruida o con las que entra en competencia".

Así, en contra del primer error, Darwin (1859, p.140) citaba el ejemplo de plantas y animales que resisten con perfecta salud cuando son trasladados a países de climas muy diferentes a los de sus países de origen; y en contra del segundo aludía, no sin cierto chauvinismo fitogeográfico, al número prodigioso de plantas que crecían en los jardines de Inglaterra, soportando perfectamente el clima allí imperante, pero sin llegar nunca a naturalizarse por no conseguir, ni competir con las plantas nativas, ni tampoco resistirse a la destrucción a la que las sometían los animales nativos (Darwin, 1859, p.69). Tal es también el caso, podría incluso haber dicho Darwin, de la distribución del ganado europeo en Sudamérica: la misma, tal como él mismo observó, no depende tanto de los rigores del clima tórrido como de la capacidad "de resistir el ataque de los insectos" que puedan tener las diferentes variedades de vacas, caballos, ovejas o cabras (Darwin, 1859, p.72 y p.195).

No se trataba, claro, de poner en duda que los factores climáticos jugasen un papel importante en la distribución, sino de reconocer que se trataba de un papel indirecto, siempre mediado por relaciones específicamente biológicas que condicionaban la distribución geográfica de las especies de un modo más inmediato que los factores climáticos (Darwin, 1859, p.175). Y es de ser notado que en este punto Darwin se distancia de Humboldt en la 
CAPONI, G. De Humboldt a Darwin: una inflexión clave en la historia ...

misma medida en que sus puntos de vista enfatizan algunas ideas ya presentes en el segundo volumen de los Principios de Geología de Charles Lyell (1832, cap.VIII): "la posibilidad de la existencia de una determinada especie en una localidad dada", nos dice Lyell (1832, p.141) ahí, "está determinada no meramente por la temperatura, la humedad, el suelo, la altura, y otras circunstancias por el estilo, sino también por la existencia o no existencia, la abundancia o la escasez, de un conjunto de otras plantas y animales en la misma región". Pero la lectura que Darwin hará de ese hecho estará también marcada por la idea de Augustin de Candolle (1820, p.384) según la cual "todas las plantas de una región (...) están en un estado de guerra las una contra las otras".

Esta imagen de una guerra de la naturaleza ya estaba presente en Bonnet (Limoges, 1976, p.71) y en la físico-teología linneana (Wilke [1760] 1972 p.119); pero en esos contextos la misma sólo aludía a las relaciones de presa-predador que contribuían a la manutención de un equilibrio natural en el que todos los seres podían preservar su lugar (Limoges, 1976, p.71). De Candolle (1820, p.385), en cambio, no solo la proyecta sobre los vegetales para indicarnos que ellos compiten entre sí por recursos como el suelo, el agua y la luz; sino que también la usa para explicar la propagación de las diferentes especies de plantas. Desde su perspectiva, la distribución de los vegetales no sólo dependía del clima sino que también obedecía a las presiones que las diferentes especies ejercían las unas sobre las otras (Bowler,1998, p.200). Cada una bregaba por preservar o conquistar su territorio en detrimento de las otras; y de su capacidad de imponerse o de simplemente persistir en esa puja, dependía su área de distribución (Limoges, 1976, p.71).

Lyell (1832, p.131), es cierto, también había citado esta tesis de De Candolle para explicar la distribución de los vegetales (Bowler,1998, p.200); pero será Darwin el que la transformará en la clave de toda la biogeografía: cada especie prospera y se propaga en la medida en que resiste la presión y el empuje de otras especies que también bregan por conseguir y sostener su precario 
CAPONI, G. De Humboldt a Darwin: una inflexión clave en la historia ...

lugar bajo el sol. En el inicio de su célebre comunicación a la Linnean Society del 30 de junio de 1858, generalizará la idea De Candolle diciendo que para éste "all nature is at war"(Darwin, 1993, p.89); y en la primera edición del Origen de las Especies, directamente dirá que De Candolle y Lyell habían "mostrado que todos los seres orgánicos están expuestos a una severa competición" (Darwin, 1859, p.62). En realidad, ni uno ni otro habían llegado tan lejos, pero ese es el corolario que Darwin sacó de la todavía algo inocente y limitada guerra de las plantas: ninguna especie, vegetal o animal, tiene un clima, un suelo o un territorio que le sea propio y peculiar; por eso cada una tendrá que luchar con las otras para conquistar y sostener su lugar en el mundo sin que ningún orden natural la preserve de su eventual extinción.

Pero lo que separa los puntos de vista de Darwin y Humboldt no es sólo ese privilegio de las conflictivas relaciones bióticas por sobre la relevancia de los factores climáticos en la explicación de la distribución geográfica de los seres vivos. Más allá de esa cuestión teórica, sus perspectivas biogeográficas también se distinguen por una cuestión que podríamos caracterizar como metodológica: mientras la historia de la naturaleza está casi totalmente ausente de la historia natural de Humboldt, ella es central en la visión desplegada por Darwin. En la biogeografía evolutiva, como en la de Buffon (Roger,1989, pp.546-547), la distribución actual de los seres no puede ser comprendida sin aludir a la historia de la tierra y de sus habitantes; y es a eso que Darwin $(1859$, p.346) se refiere cuando, en su capítulo sobre "Distribución Geográfica", nos dice que "ni la semejanza ni la desemejanza de los habitantes de varias regiones pueden explicarse totalmente por las condiciones climáticas y otras condiciones físicas".

Ahí, Darwin no está aludiendo a esos factores bióticos que hasta aquí hemos discutido. En este caso Darwin se refiere a otras dos cuestiones que también son centrales para su biogeografía: una es la existencia o inexistencia, presente o pasada, de barreras o conexiones geográficas naturales que puedan haber impedido o posibilitado migraciones (Darwin,1859, p.347); y la otra es la 
CAPONI, G. De Humboldt a Darwin: una inflexión clave en la historia ...

vinculación genealógica que puede existir entre ciertas especies que habitan una región (Darwin,1859, p.349). Para él, las notorias particularidades de los mamíferos australianos no deberían explicarse por la existencia de condiciones climáticas o ecológicas peculiares a ese continente: Australia no es el lugar natural de los marsupiales, ni tampoco tiene ninguna propensión telúrica a producirlos. Que todos los mamíferos que el hombre encontró en ese continente sean de ese tipo sólo se explica por el aislamiento en el cual esa peculiar fauna de mamíferos evolucionó y por la vinculación genealógica que existiría entre todas las especies que la componen.

Pero, no es sólo al hablar de una vinculación genealógica entre los seres vivos que estamos haciendo entrar una variable histórica ausente en la biogeografía de Humboldt: cuando citamos vicisitudes de la historia de la tierra que podrían explicar la distribución actual de los seres vivos ya nos permitimos hipótesis casi totalmente excluidas de su biogeografía. Sin negar la posibilidad de un estudio científico de la historia de la tierra, e incluso adoptando al respecto una posición catastrofista a la Cuvier bajo cuya perspectiva también podían ser explicadas las extinciones de las que daba testimonio el registro fósil (cfr. Humboldt:1805, p.23 y [1845]1977, p.52), Humboldt prefería no apelar a esas consideraciones en su biogeografía (cfr. Radl, 1931, p.262; Rehbock,1983, p.153). Así, aun habiendo observado que en la Silla de Caracas también puede encontrarse cierto vegetal, la Befaria, que crece, a doscientas leguas de distancia, en las laderas de las montañas del reino de la Nueva Granada, Humboldt, ([1802]1991a, p.347) explícitamente se recusa a preguntarse cómo esa planta pudo llegar tan lejos; y la razón de esa recusa reside en que, para él, "el primer origen de las cosas no puede ser ni un problema de historia, ni un objeto de investigación para un naturalista"(Humboldt, 1811, p.38). Desde su perspectiva, basta apuntar el hecho de que estas dos regiones hoy presentan un clima lo suficientemente frío como para posibilitar el crecimiento de esa planta (Humboldt, [1802]1991a, p.348). 
CAPONI, G. De Humboldt a Darwin: una inflexión clave en la historia ...

Pero ese hipótesis históricas non fingo de Humboldt pronto comenzaría a ser desobedecido por otros naturalistas; y aquí hay que citar otra vez a Augustin De Candolle (1820, p.383) y a Charles Lyell (1832, p.159) como "precursores" del punto de vista darwiniano. En sus Principios de Geología, este último se vuelve explícitamente contra Humboldt y afirma que, más allá de lo que ocurra en la biogeografía, en la geología "el estado de la creación animada como ahora existe" solo interesa en función de "sus relaciones con periodos antecedentes en los que sus condiciones eran diferentes"(Lyell, 1832, p.179). Pero no cabe duda que fue Edward Forbes (1846, p.350) quien, influenciado por los trabajos del propio Lyell (Bowler,1998, p.200), delineó ese enfoque histórico de la biogeografía que sería crucial para la constitución de la teoría darwiniana (Rehbock,1983, p.169 y p.187). Para él, al igual que para su seguidor Alphonse De Candolle (1855, pp.xiixiii), la distribución actual de los seres no podía ser explicada sin aludir a la historia de la tierra (Rehbock,1983, p.188); y por eso era legítimo y necesario remitirse a ella para buscar esa explicación.

Es decir: tanto para Lyell y Forbes, como para Augustin y Alphonse De Candolle, la distribución actual de los seres vivos era también una ventana hacia la historia de la naturaleza; y fue por esa ventana que Darwin se atrevió a asomarse para encontrar las claves y las respuestas de varios problemas que Humboldt reputaba insolubles. Aquí, como en el caso de la incidencia de los factores bióticos en la distribución actual de los seres, también puede decirse que, en cierto sentido, las anomalías o perplejidades de la biogeografía humboldtiana son los puntos de partida de la revolución darwiniana. Humboldt ([1802]1991a, p.345) había dicho que "por más que la razón interdiga al hombre las hipótesis sobre el origen de las cosas, no dejamos de atormentarnos por esos problemas insolubles de la distribución de los seres"; pero fue precisamente ese tormento el que condujo a Darwin hacia la formulación de una historia natural evolucionaria dentro de la cual la biogeografía constituía una pieza o un engranaje fundamental. 
CAPONI, G. De Humboldt a Darwin: una inflexión clave en la historia ...

\section{Referências bibliográficas}

Bowler, P. Historia Fontana de las ciencias ambientales.

México, Fondo de Cultura Económica, 1998.

Buffon, G. Histoire naturelle genérale et particulière III. Paris, L'Imprimiere Royale, 1749.

Buffon, G. Histoire naturelle genérale et particulière IX. Paris, L'Imprimiere Royale, 1761.

Buffon, G. Histoire naturelle genérale et particulière XIV. Paris, L'Imprimiere Royale, 1766.

Canguilhem, G. La Connaissance de la Vie. Paris, Vrin, 1965.

Caponi, G. "El viviente y su medio: antes y después de Darwin". Scientiae Studia 4 (1), p.9-43, 2006.

Cuvier, G. Tableau élémentaire de l'histoire naturelle des animaux. Paris, Baudouin, 1798.

Darwin C. "On the tendency of species to form varieties" [1858]; en D. Porter \& P. Graham (eds.), The portable Darwin. Nueva York, Penguin, 1993. [pp.86-104].

Darwin, C. On the Origin of Species. London, Murray, 1859.

De Candolle, Aug. "Géographie Botanique"; en F. Cuvier (ed.), Dictionnaire des Sciences Naturelles, Tome XVIII. Paris, Levrault, 1820 [pp.359-436].

De Candolle,, Alp. Géographie Botanique Raisonnée. Paris, Masson, 1855.

Drouin, J. L'Écologie et son histoire. Paris, Flammarion, 1993.

Flourens, P. Histoire des travaux et des idées de Buffon. Paris, Hachette, 1850.

Forbes, E. "On the connection between the distribution of the existing flora and fauna of the British Isles, and geological changes which have affected their area, especially during the epoch of the 
CAPONI, G. De Humboldt a Darwin: una inflexión clave en la historia ...

Northern Drift". Memoirs of the Geological Survey of Great Britain 1: 336-432 (with two plates), 1846.

Geoffroy Saint-Hilaire, I. "Recherches Zoologiques et physiologiques sur les variations de la taille chez les animaux et dans les races humaines". Mémoires présentes par divers savans a l'Academie Royale des Sciences de L'Institut de France 3: 503-572, 1832.

Humboldt, A. Viaje a las Regiones Equinocciales del Nuevo Mundo II. Caracas, Monte Ávila, 1991a [1802].

Humboldt, A. Viaje a las Regiones Equinocciales del Nuevo Mundo IV. Caracas, Monte Ávila, 1991b[1802].

Humboldt, A. "Essai sur le Géographie des Plantes"; in A. Humboldt \& A. Bompland: Voyage de Humboldt et Bompland XV. Paris, Lebrault \& Schoell, 1805. p.13-35.

Humboldt, A. "Essai sur 1'histoire naturalle du condor"; in A. Humboldt \& A. Bompland 1811: Voyage de Humboldt et Bompland, deuxième partie, observations de zoologie et d'anatomie comparée. Paris, Schoell \& Dufour, 1811. p.26-45.

Humboldt, A. Introducción a Cosmos. Ensayo de una descripción física del mundo [1845]; in R. Figueira (comp.) Geografía, ciencia humana. Buenos Aires, C.E.A.L, 1977. p.39-84.

Jacob, F. La lógica de lo viviente. Barcelona, Laia, 1973.

Limoges, C. La selección natural. México, Siglo XXI, 1976.

Linneo, C.: "Discurso sobre el crecimiento de la tierra habitable" [1744]; Apéndice II de Papavero, N. // Pujol LUZ, J.// LlorenteBousquets, J.: Historia de la Biología Comparada V (el Siglo de las Luces, Parte I). México, UNAM, 2001. p.135-151.

Lyell, C. Principles of Geology II. London, Murray, 1832. 
CAPONI, G. De Humboldt a Darwin: una inflexión clave en la historia ...

Papavero, N. // Teixeira, D.// Llorente-Bousquets, J. História da Biogeografía no Período Pré-Evolutivo. São Paulo, Plêiade, 1997.

Radl, E. Historia de las Teorías Biológicas II. Madrid, Biblioteca de la Revista de Occidente, 1931.

Rehbock, P. The Philosophical Naturalists: themes in early XIX century. Madison, The University of Wisconsin Press, 1983.

Roger, J. Buffon. Paris, Fayard, 1989.

Wilke, H. La police de la nature [1760]; in Linné, C. L'Équilibre de la nature (textes traduits par Bernard Jazmín, introduits et annotés par Camile Limoges). Paris, Vrin, 1972: p.103-121.

Recebido em outubro de 2006

Aceito em julho de 2007 
CAPONI, G. De Humboldt a Darwin: una inflexión clave en la historia ... 